\title{
Chemical enrichment of ICM in a hierarchical galaxy formation model including SNe Ia
}

\author{
Masahiro Nagashima ${ }^{1,2}$, Cedric G. Lacey ${ }^{1}$, Carlton M. Baugh ${ }^{1}$, \\ Carlos S. Frenk ${ }^{1}$ and Shaun Cole ${ }^{1}$ \\ ${ }^{1}$ Department of Physics, University of Durham, South Road, \\ Durham DH1 3LE, U.K. \\ ${ }^{2}$ Department of Physics, Kyoto University, Sakyo-ku, Kyoto 606-8502, Japan email: \\ masa@scphys.kyoto-u.ac.jp
}

\begin{abstract}
Metal enrichment of intracluster medium (ICM) is reanalysed in the hierarchical galaxy formation scenario. In order to investigate metal abundances of $\alpha$ and iron-peak elements, we incorporate metal production processes by type Ia supernovae (SNe Ia) as well as type II supernovae (SNe II) into the GALFORM model of semi-analytic galaxy formation. We find that a top-heavy initial mass function (IMF) of stars with a power index $x \simeq 0.35$ for starburst is required under the constraint that the IMF for quiescent disc star formation is the Kennicutt IMF. Our model shows an only mild evolution of $[\mathrm{Fe} / \mathrm{H}]$ as suggested by a recent observation.
\end{abstract}

Type Ia supernovae (SNe Ia) give us useful information on the formation and evolution of galaxies because of the delayed explosion. Furthermore, since the abundance patterns are different between SNe Ia and type II SNe (SNe II), to investigate metal abundances of various elements is quite important to understand galaxy formation. To do this, we incorporate the metal enrichment process due to SNe Ia in addition to that due to $\mathrm{SNe}$ II with the GALFORM model of semi-analytic galaxy formation (Cole et al. 2000).

The initial mass function (IMF) of stars for solar-neighbourhood stars has been considered to be a Salpeter-like bottom-heavy one, which has been confirmed by both theories and observations, including an analysis using a semi-analytic model (Nagashima \& Okamoto 2003). However, some studies on intracluster medium (ICM) based both on traditional monolithic collapse models and numerical simulations have suggested that the IMF should be rather top-heavy. Such a top-heavy IMF is recently suggested for the starburst galaxy M82 by some observations. Then, assuming the Kennicutt IMF for quiescent disc star formation and a top-heavy IMF $(x=0.35)$ for starburst here, we examine the metal abundances of ICM in a framework of the hierarchical galaxy formation. The life-time and chemical yields of SNe Ia are computed in a consistent manner with the adopted IMFs and stellar evolution.

In Figure 1, we show metal abundances of $\mathrm{O}, \mathrm{Fe}, \mathrm{Mg}$ and $\mathrm{Si}$ of ICM. The dots and crosses denote models with and without the top-heavy IMF for starburst. These models take into account the superwind, which expels gas and metals from dark haloes, and subsequent recaptures. Both models have the Kennicutt IMF for quiescent star formation. The open symbols indicate observations corrected by taking into account metallicity gradients. As clearly shown, the top-heavy IMF is required to be consistent with the observed metal abundances. We have also confirmed that another model with thermal conduction to stop gas cooling in massive haloes without superwind is also in agreement with the observation when the top-heavy IMF is adopted. 


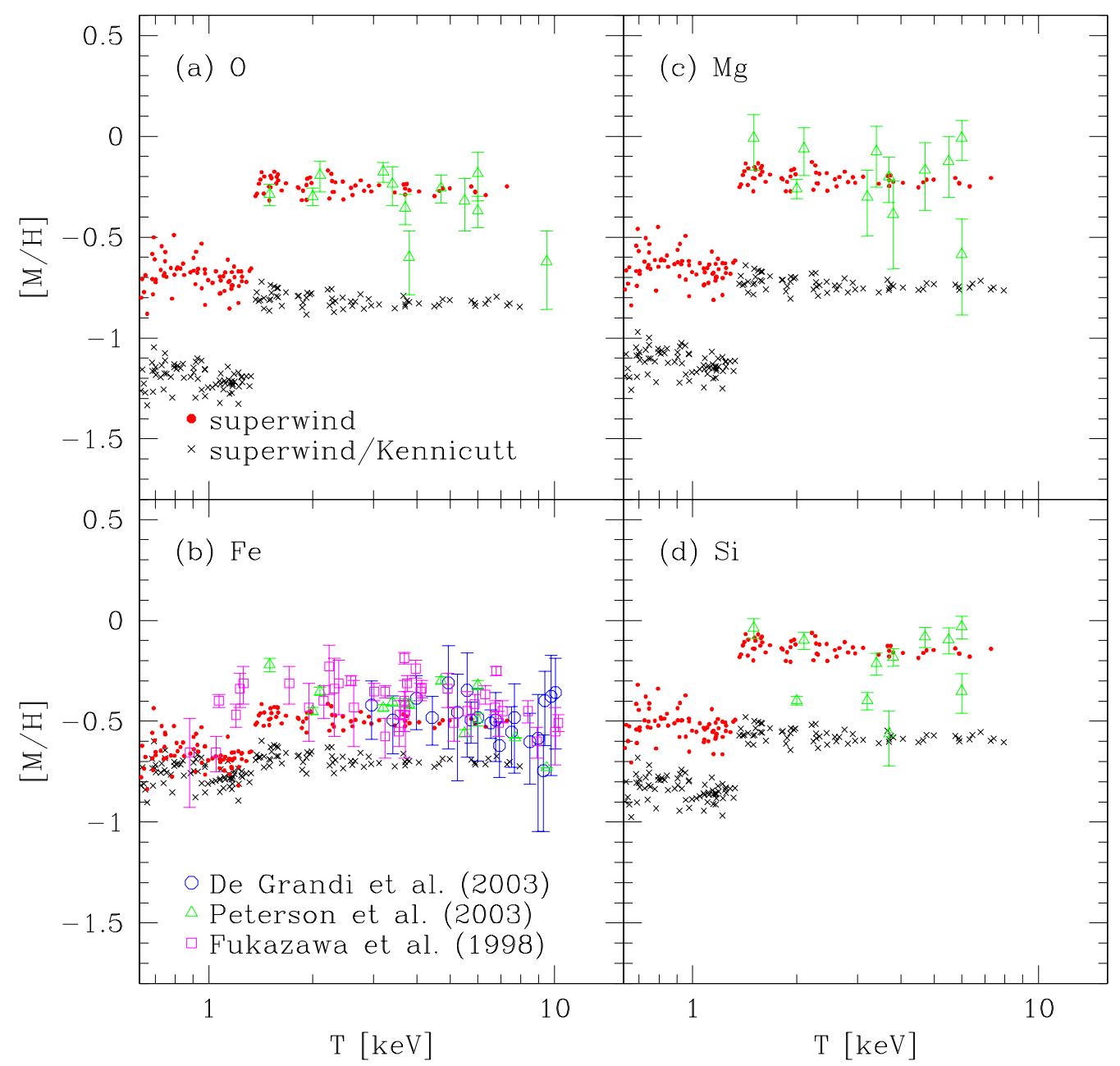

Figure 1. Metal abundances of ICM. (a) $[\mathrm{O} / \mathrm{H}]$. (b) $[\mathrm{Fe} / \mathrm{H}]$. (c) $[\mathrm{Mg} / \mathrm{H}]$. (d) $[\mathrm{Si} / \mathrm{H}]$. The dots and crosses indicate the superwind models with the top-heavy IMF and with the Kennicutt IMF for starburst. The open triangles in all panels denote the observed data by XMM-Newton (Peterson et al. 2003). The open circles and squares in panel (b) are data by BeppoSAX (De Grandi et al. 2003) and by $A S C A$ (Fukazawa et al. 1998), respectively.

Figure 2 shows redshift evolution of $[\mathrm{Fe} / \mathrm{H}]$. The iron metallicity gradually decreases toward higher redshift. The difference between $z=0$ and 1 is only $\sim 0.2$ dex. While in the observations low temperature clusters show systematically higher $[\mathrm{Fe} / \mathrm{H}]$ than high temperature ones, the model clusters do not show such a tendency. This apparent discrepancy would be solved by increasing observational data and by systematic studies of evolution of metallicity gradients.

The model with the superwind shows slightly lower $[\mathrm{Fe} / \mathrm{H}]$ than the model with thermal conduction, presumably because of its strong feedback. The difference between the two models hardly changes even at high redshift.

All of the model clusters show almost the same metallicities, metallicity ratios, and redshift evolution of metallicities, in contrast with the observations. The diversity of observed metallicities may be caused by the variety of metallicity gradients, which is presumably induced by the diversity of formation and assembly histories of the clusters. 


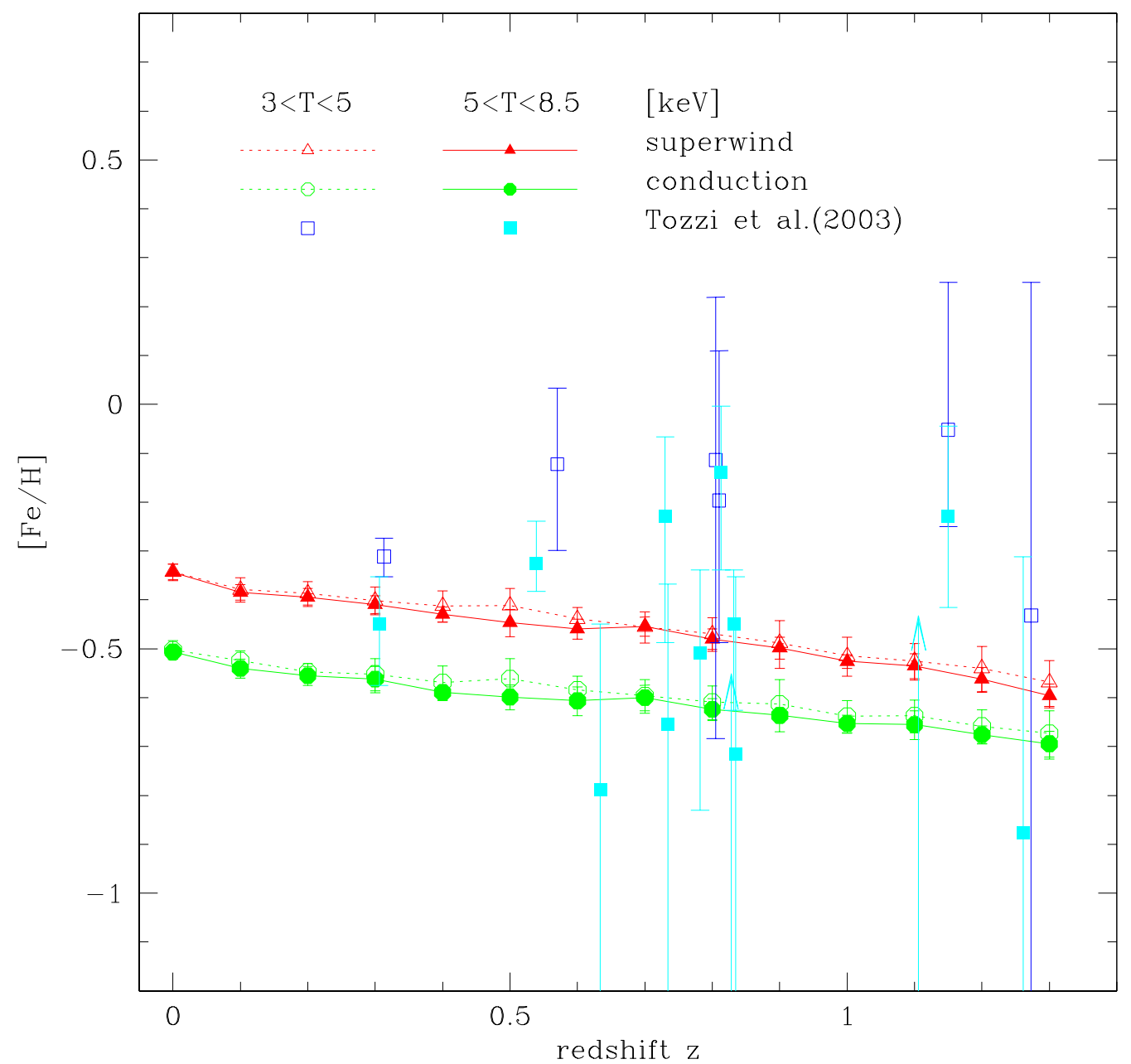

Figure 2. Redshift evolution of $[\mathrm{Fe} / \mathrm{H}]$ of ICM. The triangles and circles indicate the superwind model and the conduction model, respectively. The squares are observed data by Tozzi et al. (2003). Samples are divided into two populations, high (filled symbols) and low (open symbols) temperature clusters. Note that, for two clusters, only the upper limits of $[\mathrm{Fe} / \mathrm{H}]$, indicated by arrows, are measured.

It will provide important information to measure metallicities in the outer envelopes of clusters of galaxies.

\section{References}

Cole, S., Lacey, C. G., Baugh, C. M. \& Frenk, C. S., 2000, MNRAS, 319, 168

De Grandi, S., Ettori, S., Longhetti, M. \& Molendi S., preprint (astro-ph/0310828)

Fukazawa, Y., Makishima, K., Tamura, T., Ezawa, H., Xu, H., Ikebe, Y., Kikuchi, K. \& Ohashi T., 1998, PASJ, 50, 187

Nagashima, M. \& Okamoto, T., 2003, submitted

Peterson, J.R., Kahn, S.M., Paerels, F.B.S., Kaastra, J.S., Tamura, T., Bleeker, J.A.M., Ferrigno, C. \& Jernigan, J.G., 2003, ApJ, 590, 207

Tozzi, P., Rosati, P., Ettori, S., Borgani, S., Mainieri, V. \& Norman, C., 2003, ApJ, 593, 705 